\title{
Un problema de valores propios para un sistema parabólico periódico y aplicaciones
}

Beatriz Elena Villa

\section{Introducción y preliminares.}

En este artículo estudiaremos el sistema lineal parabólico de valores propios

$$
\begin{cases}\tilde{L}[\vec{u}]=\lambda \gamma \vec{u} & \text { en } \Omega \times \mathbb{R}, \\ \vec{u}(x, t)=\vec{u}(x, t+\ell) & \text { en } \bar{\Omega} \times \mathbb{R}, \\ \vec{u}(x, t) \equiv 0 & \text { en } \partial \Omega \times \mathbb{R}\end{cases}
$$

donde $\vec{u}=\operatorname{col}\left(u^{1}, \ldots, u^{m}\right), \ell$ es un número real positivo fijo, $\Omega \subset$ $\mathbb{R}^{N}(N \geq 1)$ es un dominio acotado cuya frontera $\partial \Omega$ es una variedad de clase $C^{2+\alpha}, \alpha \in(0,1), \tilde{L}[\vec{u}]=\operatorname{col}\left(L\left[u^{1}\right], \ldots, L\left[u^{m}\right]\right), L$ es un operador lineal de segundo orden uniformemente parabólico en $\Omega \times \mathbb{R}$ y $\gamma(x, t)=$ $\left[\gamma_{i j}(x, t)\right]$ es una matriz $m \times m$ con componentes $\gamma_{i j}(x, t)$ continuas en $\bar{\Omega} \times \mathbb{R}, \ell$-periódicas en la variable $t,(m$ es un entero positivo) y $\lambda \in \mathbb{R}$. 
Los resultados sobre (1.1) se aplicarán al estudio del sistema

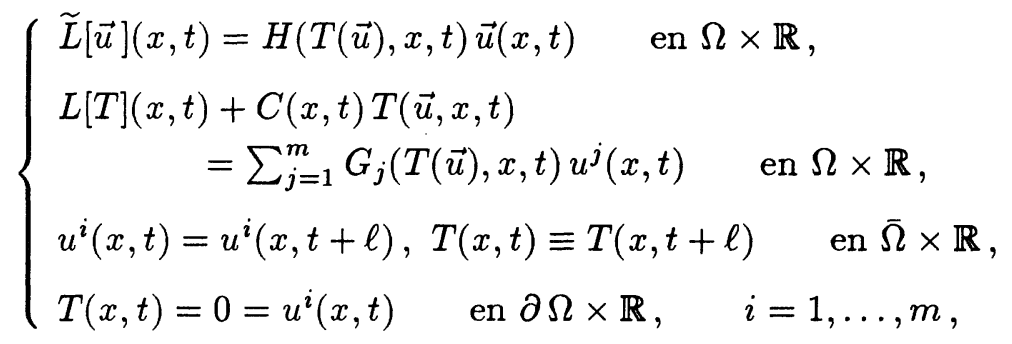

donde $H(s, x, t)=\left[H_{i j}(s, x, t)\right]$ es una matriz $m \times m$, las funciones $G_{j}, H_{i j}$ son continuas en $\mathbb{R}_{+} \times \bar{\Omega} \times \mathbb{R}, \ell$-periódicas en la variable $t \mathrm{y}$ $C(x, t)$ es continua en $\bar{\Omega} \times \mathbb{R}$ y $\ell$-periódica en $t$.

Los resultados referentes a (1.1) tienen muchas aplicaciones en la Teoría de Bifurcación.

Se conocen varias publicaciones sobre el estudio del caso escalar de (1.1) $(m=1)$. Para $L$ elíptico y autoadjunto por Manes-Micheletti [10] y por Hess-Kato para operadores no necesariamente autoadjuntos [4]. El caso parabólico fue estudiado por Laser en [7], $(\gamma=1, m=1)$.

$\mathrm{El}$ sistema (1.2) es un modelo que describe el fenómeno de reacción difusión en el interior de un reactor nuclear de fisión. Ha sido estudiado para el caso elíptico con resultados parciales por Anthony Leung y GenShun Chen en [9] y [8]. En este artículo demostraremos la existencia de soluciones clásicas positivas de este sistema, con hipótesis que se especificarán en esta misma sección. Para mayor información sobre el significado físico de las funciones que aparecen en (1.2), veánse los artículos antes mencionados.

En este artículo $J$ denotará al conjunto $J=\{1, \ldots, m\}$ y $\sigma \in$ $(\alpha, 1)$. Se introducen los siguientes espacios de Banach sobre los reales

$$
\mathbb{F}=\left\{u \in C^{\alpha, \alpha / 2}(\bar{\Omega} \times \mathbb{R}): \quad u(x, t) \text { es } \ell \text {-periódica en } t\right\}
$$

con la norma

$$
\begin{aligned}
& \|\cdot\|_{\mathbb{F}}=\|\cdot\|_{C^{\alpha, \alpha / 2}(\bar{\Omega} \times[0, \ell])}=\|\cdot\|_{\alpha}^{\bar{\Omega} \times[0, \ell]}, \\
& \mathbb{E}=\left\{u \in C^{2+\alpha, 1+\alpha / 2}(\bar{\Omega} \times \mathbb{R}): u(x, t+\ell)=u(x, t) \quad \text { en } \bar{\Omega} \times \mathbb{R}\right. \\
& u \equiv 0 \quad \text { en } \partial \Omega \times \mathbb{R}\},
\end{aligned}
$$


con la norma

$$
\begin{gathered}
\|\cdot\|_{\mathbb{E}}=\|\cdot\|_{C^{2+\alpha, 1+(\alpha / 2)(\bar{\Omega} \times[0, \ell])}}=\|\cdot\|_{2+\alpha}^{\bar{\Omega} \times[0, \ell]}, \\
\widetilde{\mathbb{F}}=\left\{u \in C^{1+\sigma, \sigma / 2}(\bar{\Omega} \times \mathbb{R}): \quad u(x, t+\ell)=u(x, t)\right\},
\end{gathered}
$$

con la norma

$$
\|\cdot\|_{\widetilde{\mathbb{F}}}=\|\cdot\|_{C^{1+\sigma, \sigma / 2}(\bar{\Omega} \times[0, \ell])}=\|\cdot\|_{1+\sigma}^{\bar{\Omega} \times[0, \ell]} .
$$

También usaremos los espacios: $\mathbb{E}^{m}=\prod_{i=1}^{m} E_{i}, E_{i}=\mathbb{E}, \mathbb{F}^{m}=$ $\prod_{i=1}^{m} F_{i}, F_{i}=\mathbb{F} ;$ y $\widehat{\mathbb{E}}=\left\{\vec{u} \in \widetilde{\mathbb{F}}^{m}: \vec{u} \equiv 0\right.$ en $\left.\partial \Omega \times \mathbb{R}\right\}$, con las respectivas normas:

$$
\begin{aligned}
\|\vec{u}\|_{\mathbb{E}^{m}} & =\max \left\{\left\|u^{i}\right\|_{\mathbb{E}}, i \in J\right\}, \\
\|\vec{u}\|_{\mathbb{T}^{m}} & =\max \left\{\left\|u^{i}\right\|_{\mathbb{F}}, i \in J\right\},
\end{aligned}
$$

y

$$
\|\vec{u}\|_{\widehat{\mathbb{E}}}=\max \left\{\left\|u^{i}\right\|_{\mathbb{\mathbb { F }}}, i \in J\right\},
$$

para todo $\vec{u}=\operatorname{col}\left(u^{1}, \ldots, u^{m}\right)$. Para mayor información acerca de las funciones Hölder-continuas veáse [7] y [3].

En $\widehat{\mathbb{E}}$ consideramos el orden natural dado por el cono positivo $\mathbb{P}=$ $\left\{\dot{\vec{u}}=\operatorname{col}\left(u^{1}, \cdots, u^{m}\right) \in \widehat{\mathbb{E}}, u^{i}(x, t) \geq 0\right.$ en $\left.\Omega \times \mathbb{R}, i \in J\right\}$. Obsérvese que el interior de $\mathbb{P}$, Int $\mathbb{P}$, es no vacío.

Un par $(\lambda, \vec{u})$ es solución clásica de (1.1) si $(\lambda, \vec{u}) \in \mathbb{R}_{+} \times \mathbb{E}^{m}$ y satisface la ecuación (1.1). En forma análoga se definen las soluciones clásicas $(T, \vec{u}) \in \mathbb{E}^{m+1}$ de $(1.2)$.

Estamos interesados en la existencia de soluciones clásicas de (1.1) y (1.2) con componentes positivas.

En lo que sigue $L$ denotará un operador de la forma

$$
L[u]=\frac{\partial u}{\partial t}-\left(\sum_{i=1}^{N} \sum_{j=1}^{N} \bar{a}_{i j} D_{i j} u+\sum_{i=1}^{N} \bar{b}_{i} D_{i} u+\bar{c}_{0} u\right),
$$

donde los coeficientes $\bar{a}_{i j}(\cdot), \bar{b}_{i}(\cdot), \bar{c}_{0}(\cdot)$ pertenecen a $\mathbb{F}, \bar{a}_{i j}=\bar{a}_{j i} \mathrm{y}$ $\bar{c}_{0} \leq 0$ en $\bar{\Omega} \times \mathbb{R}, \bar{a}_{i j} \in C^{1,0}(\bar{\Omega} \times \mathbb{R})$.

Todos los resultados de este artículo son válidos si $L$ es uniformemente elíptico en $\Omega$ y las funciones involucradas en (1.1) y (1.2) son independientes de $t$. Es decir, si $L$ es un operador de la forma

$$
L[u]=-\left(\sum_{i=1}^{N} \sum_{j=1}^{N} \tilde{a}_{i j} D_{i j} u+\sum_{i=1}^{N} \tilde{b}_{i} D_{i} u+\widetilde{c}_{0} u\right)
$$


donde las funciones $\widetilde{a}_{i j}, \widetilde{b}_{i}, \widetilde{c}_{0}$, pertenecen a $C^{\alpha}(\Omega), \widetilde{a}_{i j}=\widetilde{a}_{j i}$ y $\widetilde{c}_{0} \leq 0$ en $\Omega, \widetilde{a}_{i j} \in C^{1}(\bar{\Omega})$ y

$$
\sum_{i, j=1}^{n} \tilde{a}_{i j}(x) \xi_{i} \xi_{j} \geq M \sum_{i=1}^{N} \xi_{i}^{2}
$$

para todo $x \in \Omega,\left(\xi_{1}, \ldots, \xi_{N}\right) \in \mathbb{R}^{N}$ y algún $M>0$.

El primer resultado que presentaremos es un Teorema de Comparación, el cual es muy útil en el estudio de sistemas parabólicos y elípticos y jugará un papel muy importante en este trabajo.

Teorema 1.1. (Teorema de comparación). Supongamos que $\vec{u}, \vec{v} \in$ $C^{2,1}\left(\Omega \times \mathbb{R}, \mathbb{R}^{m}\right) \cap C^{1,0}\left(\bar{\Omega} \times \mathbb{R}, \mathbb{R}^{m}\right), \vec{u} \not \equiv \overrightarrow{0}$, satisfacen

$$
\left\{\begin{array}{l}
L\left[u^{i}\right]=\sum_{j=1}^{m} p_{i j} u^{j} \quad \text { en } \Omega \times \mathbb{R}, \quad i \in J \\
\vec{u}(x, t+\ell)=\vec{u}(x, t) \\
\left.\vec{u}\right|_{\partial \Omega \times \mathbb{R}} \equiv \overrightarrow{0}
\end{array}\right.
$$

$$
\left\{\begin{array}{l}
L\left[v^{i}\right] \geq \sum_{j=1}^{m} q_{i j} v^{j} \quad \text { en } \Omega \times \mathbb{R} \\
v^{i}(x, t+\ell)=v^{i}(x, t), \quad i \in J
\end{array}\right.
$$

donde $p_{i j}$ y $q_{i j}$ son funciones acotadas en $\bar{\Omega} \times \mathbb{R}, \ell$-periodicas en $t$. Supongamos que $v^{i} \geq 0, v^{i} \not \equiv 0$ en $\Omega \times \mathbb{R}, q_{i j} \geq p_{i j}$ en $\bar{\Omega} \times \mathbb{R}, i, j \in J$ y $q_{i j}(x, t), p_{i j}(x, t) \geq 0$ en $\bar{\Omega} \times \mathbb{R}$, para $i \neq j$. Entonces existen $\delta \in \mathbb{R}$ y $s \in J$ tales que $v^{s} \equiv \delta u^{s}, v^{j}-\delta u^{j} \geq 0$, y $p_{s j} \equiv q_{s j}$ en $\bar{\Omega} \times \mathbb{R}$, para todo $j \in J .\left(\delta=\min \left\{\sup \left\{c_{i}: v^{i}-c_{i} u^{i}>0\right.\right.\right.$ en $\left.\left.\left.\Omega \times \mathbb{R}\right\}: i \in J\right\}\right)$.

Corolario 1.1. Si suponemos las mismas hipótesis del Teorema 1.1 y suponemos que alguna componente de $\vec{u}$ toma algún valor positivo, entonces el resultado del Teorema 1.1 sigue siendo válido, aun cambiando la igualdad en (1.4) por "inferior o igual".

Adoptaremos las siguientes hipótesis sobre la matriz $\gamma$

(A1) Existe una biyección $\theta$ del conjunto $\{1, \ldots, m\}$ en sí mismo tal que

a) $\gamma_{\theta(1) k} \not \equiv 0, k=1, \ldots, m$. 
b) Para todo $j \geq 2$ existe $i \in\{1, \ldots, j-1\}$ tal que $\gamma_{\theta(j) \theta(i)} \not \equiv 0$.

(A2) Las componentes de $\gamma$ pertenecen a $\mathbb{F}$ y $\gamma_{i j} \geq 0$ en $\bar{\Omega} \times \mathbb{R}$ si $i \neq$ $j ; i, j=1, \ldots, m$.

(A3) Existe una función continua $h:[0, \ell] \rightarrow \Omega$ tal que $h(0)=h(\ell) \mathrm{y}$ para algún $k$,

$$
\int_{0}^{\ell} \gamma_{k k}(h(s), s) d s>0
$$

Observación 1.1. Por la condición (A1), si $\vec{u} \in \mathbb{P}$ es solución de (1.1) para $\lambda>0$ y una de las componentes de $\vec{u}$ es positiva en $\Omega \times \mathbb{R}$, entonces $\vec{u} \in \operatorname{Int} \mathbb{P}$. La condición (A1) se satisface por ejemplo si todas las componentes de la fila $i$ y la columna $i$ de $\gamma$ son no nulas. Por el contrario, es de notar que si todas las componentes de dos filas de $\gamma$ son nulas, excepto los elementos de la diagonal, los cuales suponemos mayores o iguales a cero y diferentes entre sí, entonces el problema (1.1) no tiene solución positiva en $\Omega \times \mathbb{R}$, para $\lambda>0$.

El resultado principal referente a (1.1) está contenido en el siguiente teorema.

Teorema 1.2. Si $\gamma$ satisface (A1)-(A3), entonces existe $\left(\lambda_{1}, \vec{u}_{1}\right) \in$ $\mathbb{R}_{+} \times \mathbb{E}^{m}, \lambda_{1}>0, \vec{u}_{1}=\operatorname{col}\left(u_{1}^{1}, \ldots, u_{1}^{m}\right)$ tal que

(i) $\left(\lambda_{1}, \vec{u}_{1}\right)$ satisface $(1.1)$.

(ii) $u_{1}^{j}>0$ en $\Omega \times \mathbb{R}, y \partial u_{1}^{j} / \partial \vec{n}<0$ en $\partial \Omega \times \mathbb{R}, j=1, \ldots, m$.

$(\partial / \partial \vec{n}$ denota diferenciación en la dirección de la normal exterior $)$,

(iii) $\lambda_{1}$ es único y simple. tesis:

Sobre las funciones $H_{i j}, G_{i}$ y $C$ adoptaremos las siguientes hipó-

(B1) $H_{i j}(T(\cdot), \cdot) \in \mathbb{F}$ si $T \in \mathbb{F}, H_{i j}$ es uniformemente acotada en $\mathbb{R}_{+} \times$ $\Omega \times[0, \ell]$ y la matriz $H(0, \cdot)$ satisface (A1)-(A3).

(B2) a) $C \in \mathbb{F}$, las funciones $G_{i}(s, x, t)$ y $\partial G_{i}(s, x, t) / \partial s$ son continuas, $G_{i}(T(\cdot), \cdot)$ y $\partial G_{i}(T(\cdot), \cdot) / \partial s \in \mathbb{F}$ si $T \in \mathbb{F}$ y $G_{i}(\cdot, x, t)$ es de- 
creciente en $\mathbb{R}_{+}$, para todo $(x, t) \in \bar{\Omega} \times[0, \ell], i=1, \ldots, m$.

b) Existen $m$ funciones $g_{i} \in \mathbb{F}$ tales que $0<g_{i}(x, t) \leq G_{i}(T, x, t)$ para todo $(T, x, t) \in \mathbb{R}_{+} \times \bar{\Omega} \times[0, \ell], i=1, \ldots, m$.

Sea $\widehat{\lambda_{0}}$ el valor propio de $(1.1)$ para $\gamma \equiv H(0, \cdot)$. En las hipótesis siguientes consideramos dos posibilidades para $\widehat{\lambda_{0}}$ :

(B3) a) $\widehat{\lambda_{0}}>1$ y existen dos números reales $a_{0}, T_{0}$ tales que $a_{0}>\widehat{\lambda}_{0} \mathrm{y}$ $H_{i j}(T, x, t) \geq a_{0} H_{i j}(0, x, t)$, para todo $T \geq T_{0}$ y $(x, t) \in \bar{\Omega} \times \mathbb{R}$.

b) Para algún $k \in\{1, \ldots, m\}, H_{k k}(0, \cdot)$ satisface (A3) y $H_{k k}$ $(T, x, t) \geq H_{k k}(0, x, t)$, para todo $(x, t) \in \Omega \times \mathbb{R}$ y $T>0$.

(B4) a) $0<\widehat{\lambda_{0}}<1$ y existen dos números reales $a_{0}^{*}$ y $T_{0}^{*}$ tales que $0<a_{0}^{*}<\widehat{\lambda_{0}}$ y $H_{i j}(T, x, t)<a_{0}^{*} H_{i j}(0, x, t)$, para todo $T \geq T_{0}^{*} \mathrm{y}$ $(x, t) \in \bar{\Omega} \times \mathbb{R}$.

b) Existe un conjunto compacto $D \subset \Omega$ tal que $H_{i j}(T, x, t)=0$ para todo $T \geq 0, t \in \mathbb{R}$ y $x \in(\bar{\Omega} / D)$.

En algunas situaciones supondremos que $\Omega$ satisface la condición:

$\left(C^{*}\right)$ Existe una sucesión $\left\{D_{k}\right\}_{k=1}^{\infty}$ de subdominios de $\mathbb{R}^{N}$, con frontera de clase $C^{2+\alpha}$ tales que $\bar{D}_{k} \subset D_{k+1} \subset \bar{D}_{k+1} \subset \Omega$, para todo $k$ y $\bigcup_{k=1}^{\infty} D_{k}=\Omega$.

Teorema 1.3. a) Supongamos que se satisfacen (B1)-(B3) y que $\Omega$ satisface $\left(C^{*}\right)$. Entonces $(1.2)$ admite una solución $\left(T, \vec{u}_{0}\right) \in \mathbb{E}^{m+1}, \vec{u}_{0}=$ $\operatorname{col}\left(u_{0}^{1}, \ldots, u_{0}^{m}\right)$ tal que $T(x, t)>0$ y $u_{0}^{i}(x, t)>0$ en $\Omega \times \mathbb{R}, i=$ $1, \ldots, m$.

b) Si se satisfacen (B1), (B2) y (B4) obtenemos el mismo resultado de la parte a).

OBSERVACIÓN 1.2. Todos los resultados permanecen válidos si suponemos que $\widetilde{L}(\vec{u})=\left(L_{1}\left(u^{1}\right), \ldots, L_{m}\left(u^{m}\right)\right), \vec{u}=\operatorname{col}\left(u^{1}, \ldots, u^{m}\right)$, donde $L_{k}$ es uniformemente parabólico en $\Omega \times \mathbb{R}$ para $k=1, \ldots, m$ y tiene la forma

$$
L_{k}[u]=\frac{\partial u}{\partial t}-\left(\sum_{i=1}^{N} \sum_{j=1}^{N} a_{i j}^{k} D_{i j} u+\sum_{i=1}^{N} b_{i}^{k} D_{i} u+c_{0}^{k} u\right),
$$


y sus coeficientes satisfacen las condiciones antes impuestas para el operador $L$.

\section{Resultados principales.}

En esta sección demostraremos los teoremas 1.1 y 1.2. Respecto al sistema (1.1), consideraremos primero, en la Proposición 1, el caso especial en el cual $\gamma$ satisface (A1) y (A2) y las componentes de su diagonal son mayores o iguales que cero y no nulas.

En la demostración del Teorema 1.1 utilizaremos el siguiente lema:

Lema 2.1. Si $u, v \in C^{2,1}(\Omega \times \mathbb{R}) \bigcap C^{1,0}(\bar{\Omega} \times \mathbb{R}), v(x, t)>0, u(x, t+l) \equiv$ $u(x, t), v(x, t+\ell) \equiv v(x, t)$, en $\bar{\Omega} \times \mathbb{R}, u(x, t) \equiv 0$ en $\partial \Omega \times \mathbb{R}$ y además $\partial v(\bar{x}, \bar{t}) / \partial \vec{n}<0$ para todo $(\bar{x}, \bar{t}) \in \partial \Omega \times \mathbb{R}$, para el cual $v(\bar{x}, \bar{t})=0$, entonces existe un número real $a>0$ tal que $v(x, t)>a u(x, t)$ para todo $(x, t) \in \Omega \times \mathbb{R}$.

Demostración. Supongamos que el lema no es cierto. Entonces podemos construir una sucesión $\left\{\left(c_{n}, x_{n}, t_{n}\right)\right\}_{n=1}^{\infty}$, con $c_{n}>0 \mathrm{y}\left(x_{n}, t_{n}\right) \in$ $\bar{\Omega} \times \mathbb{R}$ tales que $c_{n} \rightarrow 0 \mathrm{y}\left(v-c_{n} u\right)\left(x_{n}, t_{n}\right)=0$, para todo entero positivo $n$. Sin pérdida de generalidad suponemos que $\left(x_{n}, t_{n}\right) \rightarrow(\bar{x}, \bar{t})$, para un $(\bar{x}, \bar{t}) \in \bar{\Omega} \times[0, \ell]$. Además $0=\lim _{n \rightarrow \infty}\left(v-c_{n} u\right)\left(x_{n}, t_{n}\right)=v(\bar{x}, \bar{t})$, implica que $(\bar{x}, \bar{t}) \in \partial \Omega \times \mathbb{R}$. Sea $z_{n}$ el punto de $\partial \Omega$ más cercano a $x_{n}$. De nuestras hipótesis sobre $u$ y $v$ y del Teorema del Valor Medio obtenemos

$$
\begin{aligned}
0 & \leq\left(v-c_{n} u\right)\left(z_{n}, t_{n}\right)-\left(v-c_{n} u\right)\left(x_{n}, t_{n}\right) \\
& =\left\langle\left(\nabla_{x} v-c_{n} \nabla_{x} u\right)\left(\xi_{n}, t_{n}\right), z_{n}-x_{n}\right\rangle
\end{aligned}
$$

donde $\xi_{n} \in \Omega$ está en el segmento que une $x_{n} \operatorname{con} z_{n},\langle\cdot, \cdot\rangle$ es el producto interno en $\mathbb{R}^{N}$ y $\nabla_{x}$ es el gradiente respecto a $x$. Entonces,

$$
\left\langle\left(\nabla_{x} v-c_{n} \nabla_{x} u\right)\left(\xi_{n}, t_{n}\right), \quad \vec{n}\left(z_{n}, t_{n}\right)\right\rangle \geq 0 .
$$

$\left(\vec{n}\left(z_{n}, t_{n}\right)\right.$ es la normal unitaria hacia el exterior a $\partial \Omega \times \mathbb{R}$ en $\left.\left(z_{n}, t_{n}\right)\right)$. $\mathrm{Si}$ hacemos tender $n$ a infinito en la última desigualdad obtenemos $\partial v(\bar{x}, \bar{t}) / \partial \vec{n} \geq 0$, lo cual contradice una de nuestras hipótesis.

Demostración Del TeOrema 1.1. Sea $k>0$ una constante suficientemente grande tal que $p_{i i}+k>0$ y $q_{i i}+k>0$ en $\bar{\Omega} \times \mathbb{R}, i=1, \ldots, m$. 
De las hipótesis, obtenemos

$$
L\left[v^{i}\right]+k v^{i}=\left(q_{i i}+k\right) v^{i}+\sum_{\substack{j=1 \\ j \neq i}}^{m} q_{i j} v^{j} \geq 0 \quad \text { en } \Omega \times \mathbb{R},
$$

$v^{i} \geq 0$ y $v^{i} \not \equiv 0$ en $\bar{\Omega} \times \mathbb{R}$. Entonces, por el Principio del Máximo para ecuaciones parabólicas [12], tenemos que

$$
v^{i}>0 \quad \text { en } \Omega \times \mathbb{R}, \quad \text { y } \quad \frac{\partial v^{i}}{\partial \vec{n}}(\bar{x}, \bar{t})<0
$$

si $v^{i}(\bar{x}, \bar{t})=0$, y $(\bar{x}, \bar{t}) \in \partial \Omega \times \mathbb{R}$.

Sea $\delta_{i}=\sup \left\{a_{i}: v^{i}-a_{i} u^{i}>0\right.$ en $\left.\Omega \times \mathbb{R}\right\}$. Por (2.1) y el Lema $2.1,0<\delta_{i} \leq \infty, i=1, \ldots, m$. Por otra parte podemos suponer sin pérdida de generalidad que alguna de las componentes de $\vec{u}$ toma un valor positivo. Entonces, si $\delta_{j} \equiv \min \left\{\delta_{1}, \ldots, \delta_{m}\right\}$, se tiene que

$$
0<\delta_{j}<\infty \quad \text { y } \quad v^{i}-\delta_{j} u^{i} \geq 0 \quad \text { en } \Omega \times \mathbb{R}, \quad i=1, \ldots, m .
$$

Más aún, probaremos que $v^{j}-\delta_{j} u^{j} \equiv 0$ en $\bar{\Omega} \times \mathbb{R}$. Por hipótesis tenemos

$$
\begin{aligned}
(L+k)\left(v^{j}-\delta_{j} u^{j}\right) \geq( & \left.k+p_{j j}\right)\left(v^{j}-\delta_{j} u^{j}\right) \\
& +\sum_{\substack{i=1 \\
i \neq j}}^{m} p_{j i}\left(v^{i}-\delta_{j} u^{i}\right) \\
& +\sum_{i=1}^{m}\left(q_{j i}-p_{j i}\right) v^{i} \geq 0 \quad \text { en } \Omega \times \mathbb{R} .
\end{aligned}
$$

Por esta desigualdad, el Lema 2.1 y la definición de $\delta_{j}$, existe un punto $\left(x_{0}, t_{0}\right) \in \Omega \times \mathbb{R}$ tal que $\left(v^{j}-\delta_{j} u^{j}\right)\left(x_{0}, t_{0}\right)=0$. Finalmente por (2.2) y el Principio del Máximo, concluímos que $v^{j}-\delta_{j} u^{j} \equiv 0 \mathrm{y}$ $p_{j i}=q_{j i}$ en $\Omega \times \mathbb{R}$, para $i=1, \ldots, m$.

El próximo lema se utilizará en lo que sigue.

Lema 2.2. Para cada $f \in \mathbb{F}$, existe una única función $u \in \mathbb{E}$ tal que $L[u] \equiv f$ en $\Omega \times \mathbb{R}$. Además existen dos constantes $k_{1}, k_{2}>0$ tales que 
a) $\|u\|_{\mathbb{E}} \leq k_{1}\|L[u]\|_{\mathbb{F}}$, para todo $u \in \mathbb{E}$,

b) $\|u\|_{C^{1+\sigma, \sigma / 2}(\bar{\Omega} \times[0, \ell])} \leq k_{2}\|L[u]\|_{\infty}$ para todo $u \in \mathbb{E}$,

$\left(k_{2}\right.$ depende de $\left.\sigma \in(\alpha, 1)\right)(c f .[11],[13])$

En la demostración del Teorema 1.2 utilizaremos la siguiente proposición.

Proposición 2.1. Si la matriz $\gamma$ satisface (A1) y (A2) y $\gamma_{i i}(x, t) \geq 0$ y $\gamma_{i i} \not \equiv 0$ en $\bar{\Omega} \times \mathbb{R}$ para todo $i=1, \ldots, m$, entonces existe $\left(\lambda_{0}, \vec{u}_{0}\right) \in$ $\mathbb{R}_{+} \times \mathbb{E}^{m}$ tal que $\lambda_{0}$ es único $y$

i) $\left(\lambda_{0}, \vec{u}_{0}\right)$ es solución de (1.1),

ii) $\vec{u}_{0} \in \mathbb{E}^{m} \cap \operatorname{Int} \mathbb{P}$ y $\partial u_{0}^{j} / \partial \vec{n}<0$ en $\partial \Omega \times \mathbb{R}, j=1, \ldots, m$.

iii) $\lambda_{0}$ es un valor propio simple.

Demostración. Sea $S: \mathbb{F}^{m} \rightarrow \mathbb{F}^{m}$ el operador lineal completamente continuo y positivo definido así:

$$
\begin{array}{r}
S\left(\operatorname{col}\left(u^{1}, \ldots, u^{m}\right)\right)=\operatorname{col}\left(J^{*} L^{-1}\left(\sum_{j=1}^{m} \gamma_{1 j} u^{j}\right), \ldots,\right. \\
\left.J^{*} L^{-1}\left(\sum_{j=1}^{m} \gamma_{m j} u^{j}\right)\right)
\end{array}
$$

donde $J^{*}: \mathbb{E} \rightarrow \mathbb{F}$ es la inyección natural, la cual es compacta, [3].

Sean $\vec{z}=\operatorname{col}\left(L^{-1}(1), \ldots, L^{-1}(1)\right)$ y $\vec{v}=(\widetilde{L})^{-1}(\gamma \vec{z})=S(\vec{z})$. Con el mismo argumento usado en el Teorema 1.1, obtenemos

$$
\begin{aligned}
& S(\vec{z})-\delta \vec{z} \in \mathbb{P}, \\
& \delta=\min \left\{\sup \left\{c_{i}: \quad v^{i}-c_{i} z^{i}>0 \text { en } \Omega \times \mathbb{R}\right\}: i \in J\right\} .
\end{aligned}
$$

Por (2.3) y un resultado muy conocido de la teoría de operadores positivos ( $c f$. [5, Teorema 2.5]), existen $\vec{u}_{0} \in \mathbb{P}, \vec{u}_{0} \not \equiv \overrightarrow{0}$ y un número real $\lambda_{1}>\delta$ tales que $S\left(\vec{u}_{0}\right)=\lambda_{1} \vec{u}_{0}$, esto es, $\widetilde{L}\left[\vec{u}_{0}\right]=\lambda_{0} \gamma \vec{u}_{0},\left(\lambda_{0}=1 / \lambda_{1}\right)$ y $\left(\lambda_{0}, \vec{u}_{0}\right)$ satisface (i) y (ii). 
La unicidad de $\lambda_{0}$ se sigue directamente del Teorema 1.1. Utilizaremos también este teorema para demostrar que $\lambda_{0}$ es simple. Sea $\vec{w}=\operatorname{col}\left(w^{1}, \ldots, w^{m}\right), \vec{w} \not \equiv \overrightarrow{0}$ tal que $\widetilde{L}[\vec{w}]=\lambda_{0} \gamma \vec{w}$. Podemos suponer que alguna componente de $\vec{w}$ toma un valor positivo en $\Omega \times \mathbb{R}$. Entonces, por el Teorema 1.1, existe $\delta^{*}>0$ tal que $u_{0}^{j}-\delta^{*} w^{j} \equiv 0$, para ciertos $j \in J$ y $u_{0}^{i}-\delta^{*} w^{i} \geq 0$ en $\Omega \times \mathbb{R}$, para todo $i \in J$. Del Principio del Máximo y la.Observación 1.1 se deduce que $\vec{u}_{0}-\delta^{*} \vec{w} \equiv \overrightarrow{0}$ en $\Omega \times \mathbb{R}$.

Para demostrar que la multiplicidad algebraica de $\lambda_{0}$ es uno, supongamos que $\vec{v}_{0}=\operatorname{col}\left(v_{0}^{1}, \ldots, v_{0}^{m}\right) \in \widehat{\mathbb{E}}, \vec{v}_{0} \not \equiv \overrightarrow{0}$, satisface

$$
\left(S-\frac{1}{\lambda_{0}} I\right)^{2} \vec{v}_{0}=0
$$

( $I$ es la matriz identidad en $\widehat{\mathbb{E}})$.

Entonces $\left(S-\lambda_{1} I\right) \vec{v}_{0}=-k^{*} \vec{u}_{0}$, para una constante $k^{*}$ que podemos suponer positiva. Sea $\widehat{\delta}>0$ suficientemente grande tal que

$$
w_{0}^{i}=\widehat{\delta} u_{0}^{i}+v_{0}^{i}>0 \quad \text { en } \Omega \times \mathbb{R}, \text { para todo } i \in J .
$$

Por un simple cálculo se obtiene

$$
L\left[w_{0}^{i}\right] \geq \lambda_{0} \sum_{j=1}^{m} \gamma_{i j} w_{0}^{j} \quad \text { en } \Omega \times \mathbb{R}, i \in J .
$$

Entonces, por el Teorema 1.1, existen $s \in J$ y $a \in \mathbb{R}_{+}$tales que

$$
w_{0}^{s}=a u_{0}^{s}, \quad w_{0}^{j}-a u_{0}^{j} \geq 0 \quad \text { en } \Omega \times \mathbb{R}, \quad j=1, \ldots, m .
$$

Por la identidad

$$
0=L\left(w_{0}^{s}-a u_{0}^{s}\right)=\lambda_{0} \sum_{j=1}^{m} \gamma_{s j}\left(w_{0}^{j}+\left(\lambda_{0} k^{*}-a\right) u_{0}^{j}\right) \quad \text { en } \Omega \times \mathbb{R},
$$

y (2.4), existe un punto $\left(x_{0}, t_{0}\right) \in \Omega \times \mathbb{R}$ tal que

$$
\left(w_{0}^{s}+\left(\lambda_{0} k^{*}-a\right) u_{0}^{s}\right)\left(x_{0}, t_{0}\right)=0 .
$$

Puesto que

$$
L\left[w_{0}^{s}+\left(\lambda_{0} k^{*}-a\right) u_{0}^{s}\right]=\lambda_{0} k^{*}\left(\lambda_{0} k^{*}-a\right) u_{0}^{s} \geq 0 \quad \text { en } \Omega \times \mathbb{R},
$$


por el Principio del Máximo obtenemos que $w_{0}^{s}+\left(\lambda_{0} k^{*}-a\right) u_{0}^{s} \equiv 0$ en $\Omega \times \mathbb{R}$, y por $(2.4), k^{*}=0$.

En el resto de esta sección supondremos que $\gamma$ satisface (A1)-(A3) y $k \in\{1, \ldots, m\}$ es el entero positivo tal que $\gamma_{k k}$ satisface (1.6). Denotaremos con $\gamma^{+} \equiv\left[\gamma_{i j}^{+}\right]$y $\gamma^{-} \equiv\left[\gamma_{i j}^{-}\right]$. Es claro que si $i \neq j, \quad \gamma_{i j}^{+}=\gamma_{i j}$ y $\gamma^{-}$es una matriz diagonal. Para cada $s \in(0,1]$, denotaremos con $\rho_{s}=\left[\rho_{i j}^{s}\right]$, donde

$$
\rho_{i j}^{s}=s \gamma_{i j}^{+} \quad \text { si } i \neq j \text { ó } i=j \neq k, \quad \rho_{k k}^{s}=\gamma_{k k}^{+} .
$$

Por la Proposición 2.1, para cada $s \in(0,1], \lambda>0$ existe $\left(B_{s}(\lambda), \vec{u}_{s \lambda}\right) \in \mathbb{R}_{+} \times\left(\mathbb{E}^{m} \bigcap \operatorname{Int} \mathbb{P}\right)$ tal que

$$
\widetilde{L}\left[\vec{u}_{s \lambda}\right]+\lambda \gamma^{-}\left[\vec{u}_{s \lambda}\right]=B_{s}(\lambda) \rho_{s}\left[\vec{u}_{s \lambda}\right] \quad \text { en } \Omega \times \mathbb{R} .
$$

Claramente, (1.1) tiene solución si y sólo si $B_{1}(\lambda)=\lambda$ para algún $\lambda>0$. Por el Teorema 1.1, para $s$ fijo, $B_{s}(\lambda)$ es estrictamente creciente en $\mathbb{R}_{+}$y, para $\lambda$ fijo, $B_{s}(\lambda)$ es estrictamente decreciente.

El siguiente resultado se utilizará en el resto de esta sección.

Lema 2.3. Si $\gamma$ satisface (A1)-(A3), entonces $B_{s}(\lambda)$ es continua en $(0,1] \times \mathbb{R}_{+}$.

Demostración. Sean $a, b>0, \lambda^{*} \in[0, a], s^{*} \in[b, 1]$ y $\left\{\left(s_{n}, \lambda_{n}\right)\right\}_{n=1}^{\infty}$ una sucesión en $[b, 1] \times[0, a]$ tal que $\left(s_{n}, \lambda_{n}\right) \rightarrow\left(s^{*}, \lambda^{*}\right)$. Podemos suponer que $B_{s_{n}}\left(\lambda_{n}\right) \rightarrow d \in\left[0, B_{b}(a)\right]$. Para cada $n$, existe $\vec{u}_{n}=$ $\operatorname{col}\left(u_{n}^{1}, \ldots, u_{n}^{m}\right) \in \mathbb{E}^{m} \cap \operatorname{Int} \mathbb{P}$ tal que.

$$
\left\|\vec{u}_{n}\right\|_{\infty} \equiv \max \left\{\left\|u_{n}^{i}\right\|_{\infty}: i=1, \ldots, m\right\}=1
$$

y

$$
(\widetilde{L})\left[\vec{u}_{n}\right]+\lambda_{n} \gamma^{-} \vec{u}_{n}=B_{s_{n}}\left(\lambda_{n}\right) \rho_{s_{n}} \vec{u}_{n} \text { en } \Omega \times \mathbb{R} .
$$

Por el Lema 2.2 la sucesión $\left\{u_{n}^{i}\right\}$ es acotada en $\widetilde{\mathbb{F}}$ y puesto que la inyección natural $J^{*}: \widetilde{\mathbb{F}} \rightarrow \mathbb{F}$ es compacta, (para una subsucesión) $u_{n}^{i} \rightarrow$ $v^{i} \in \mathbb{F}$, en la norma de $\mathbb{F}, i=1, \ldots, m, \vec{v}=\operatorname{col}\left(v^{1}, \ldots, v^{m}\right),\|\vec{v}\|_{\infty}=1$. Entonces

$$
\begin{aligned}
\vec{v} & =\lim _{n \rightarrow \infty} \vec{u}_{n}=\lim _{n \rightarrow \infty}(\widetilde{L})^{-1}\left(-\lambda_{n} \gamma^{-} \vec{u}_{n}+B_{s_{n}}\left(\lambda_{n}\right) \rho_{s_{n}} \vec{u}_{n}\right) \\
& =(\widetilde{L})^{-1}\left(-\lambda^{*} \gamma^{-} \vec{v}+d \rho_{s^{*}} \vec{v}\right) .
\end{aligned}
$$


Finalmente para $\left(s^{*}, \lambda^{*}\right)$, existe $\vec{u}_{s^{*} \lambda^{*}} \in \mathbb{E}^{m} \bigcap \operatorname{Int} \mathbb{P}$ tal que

$$
(\widetilde{L})\left(\vec{u}_{s^{*} \lambda^{*}}\right)=-\lambda^{*} \gamma^{-} \vec{u}_{s^{*} \lambda^{*}}+B_{s^{*}}\left(\lambda^{*}\right) \rho_{s^{*}} \vec{u}_{s^{*} \lambda^{*}} \quad \text { en } \Omega \times \mathbb{R} .
$$

De (2.6), (2.7) y el Teorema 1.1 se deduce que $B_{s^{*}}\left(\lambda^{*}\right)=d$.

Lema 2.4. Para s fijo, $B_{s}(\lambda)$ tiene por lo menos un punto fijo.

Demostración. Sea $s \in(0,1]$ fijo. Puesto que $B_{s}(\lambda)$ es continua y $B_{s}(0)>0$, es suficiente probar que $B_{s}\left(\lambda^{*}\right)<\lambda^{*}$ para algún $\lambda^{*}>0$.

Sea $G \in \mathbb{R}^{N}$ una bola abierta con centro 0 , tal que $y+h(r) \in \Omega$ para todo $y \in G$ y $r \in[0, \ell]$ y

$$
\int_{0}^{\ell} \gamma_{k k}(y+h(r), r) d s>0
$$

para todo $y \in G$.

Sea $\phi: \mathbb{R}^{N} \rightarrow[0,1], \phi \not \equiv 0$ una función de clase $C^{\infty}$ con soporte compacto contenido en $G$. Es claro que

$$
\int_{G \times[0, \ell]} \gamma_{k k}(y+h(r), r) \phi^{2}>0 .
$$

Introducimos la siguiente notación

$$
\begin{aligned}
h(r) & =\left(h_{1}(r), \ldots, h_{N}(r)\right), \\
a_{i j}(y, r) & =\bar{a}_{i j}(y+h(r), r), \\
b_{i}(y, r) & =\bar{b}_{i}(y+h(r), r), \\
c_{0}(y, r) & =\bar{c}_{0}(y+h(r), r), \\
\bar{\psi}_{\lambda}^{j}(x, t) & =\log \left(u_{s \lambda}^{j}(x, t)\right), \\
\psi_{\lambda}^{j}(y, r) & =\bar{\psi}_{\lambda}^{j}(y+h(r), r), \\
D_{i} \psi_{\lambda}^{k} & =D_{y_{i}} \psi_{\lambda}^{k} \\
D_{i j} \psi_{\lambda}^{k} & =D_{y_{i} y_{j}} \psi_{\lambda}^{k} .
\end{aligned}
$$

Remplazando $\bar{\psi}_{\lambda}^{k}$ y sus derivadas en (2.5) obtenemos

$$
\frac{\partial}{\partial t} \bar{\psi}_{\lambda}^{k}(x, t)-\sum_{i, j=1}^{N} \bar{a}_{i j} D_{x_{i} x_{j}} \bar{\psi}_{\lambda}^{k}-\sum_{i, j=1}^{N} \bar{a}_{i j} D_{x_{i}} \bar{\psi}_{\lambda}^{k} D_{x_{j}} \bar{\psi}_{\lambda}^{k}-
$$




$$
-\sum_{i=1}^{N} \bar{b}_{i} D_{x_{i}} \bar{\psi}_{\lambda}^{k} \geq \bar{c}_{0}(x, t)-\lambda \bar{\gamma}_{k k}(x, t)+B_{s}(\lambda) \gamma_{k k}^{+}(x, t)
$$

Cambiando de variables, multiplicando por $\phi^{2}$ e integrando sobre $D=G \times[0, \ell]$, obtenemos

$$
\begin{aligned}
\int_{D}\left(\frac{\partial \psi_{\lambda}}{\partial r}\right. & -\sum_{i=1}^{N} D_{i} \psi_{\lambda}^{k} h_{i}^{\prime}-\sum_{i, j=1}^{N} a_{i j} D_{i j} \psi_{\lambda}^{k} \\
& \left.-\sum_{i, j=1}^{N} a_{i j} D_{i} \psi_{\lambda}^{k} D_{j} \psi_{\lambda}^{k}-\sum_{i=1}^{N} b_{i} D_{i} \psi_{\lambda}^{k}\right) \phi^{2} \\
& \geq \int_{D}\left[c_{0}-\lambda \gamma_{k k}^{-}(y+h(r), r)+B_{s}(\lambda) \gamma_{k k}^{+}(y+h(r), r)\right] \phi^{2}
\end{aligned}
$$

Puesto que $\int_{D} \frac{\partial \psi_{\lambda}^{k}}{\partial r} \phi^{2} d r d y=0$, integrando por partes obtenemos de (2.9)

$$
\begin{aligned}
\int_{D}\left(B_{s}(\lambda) \gamma_{k k}^{+}-\lambda \gamma_{k k}^{-}\right)(y+h(r), r) \phi^{2}(y, r) \\
\leq-\int_{D}\left(\left\langle\phi \nabla_{y} \psi_{\lambda}^{k}, A\left(\phi \nabla_{y} \psi_{\lambda}^{k}\right)+\vec{d}\right\rangle+c_{0} \phi^{2}\right)
\end{aligned}
$$

donde $\vec{d}=\left(d_{1}, \ldots, d_{m}\right)$, con

$$
d_{j}=-\sum_{i=1}^{N}\left(D_{i} a_{i j} \phi+2 a_{i j} D_{i} \phi\right)+b_{j} \phi+h_{j}^{\prime} \phi
$$

$A$ es la matriz $A=\left[a_{i j}\right], \nabla_{y}$ es el gradiente respecto a $y, \mathrm{y}\langle\cdot, \cdot\rangle$ el producto escalar en $\mathbb{R}^{N}$. Observemos que $\vec{d}$ es independiente de $\psi_{\lambda}^{k}$.

Por ser $A$ simétrica y $L$ uniformemente parabólico, tenemos que para todo vector $\vec{B}$

$$
\begin{aligned}
\langle\vec{B}, A \overrightarrow{\vec{B}}+\vec{d}\rangle=\langle\vec{B} & \left.+\frac{1}{2} A^{-1} \vec{d}, A\left(\vec{B}+\frac{1}{2} A^{-1} \vec{d}\right)\right\rangle \\
& -\frac{1}{4}\left\langle A^{-1} \vec{d}, \vec{d}\right\rangle \geq-\frac{1}{4}\left\langle A^{-1} \vec{d}, \vec{d}\right\rangle .
\end{aligned}
$$


Entonces, de (2.10), obtenemos

$$
B_{s}(\lambda) \leq a+\frac{\lambda \int_{D} \gamma_{k k}^{-}(y+h(r), r) \phi^{2}}{\int_{D} \gamma_{k k}^{+}(y+h(r), r) \phi^{2}},
$$

donde

$$
a=\frac{\int_{D}\left[\frac{1}{4}\left\langle A^{-1} \vec{d}, \vec{d}\right\rangle-c_{0} \phi^{2}\right]}{\int_{D} \gamma_{k k}^{+}(y+h(r), r) \phi^{2}} .
$$

De (2.8), deducimos que existe $\lambda^{*}$ tal que para todo $\lambda>\lambda^{*}$,

$$
\frac{\lambda\left(\int_{D} \gamma_{k k}(y+h(r), r) \phi^{2}\right)}{\int_{D} \gamma_{k k}^{+}(y+h(r), r) \phi^{2}}>a,
$$

y por $(2.11), B_{s}(\lambda)<\lambda$ para todo $\lambda \geq \lambda^{*}$, y por tanto, $B_{s}(\lambda)$ tiene un punto fijo menor que $\lambda^{*}$.

Del lema anterior se desprende la existencia de soluciones de (1.1). $\mathrm{El}$ resto de esta sección lo dedicamos a probar la unicidad de los valores propios de (1.1) con función propia con componentes positivas. Para cada $s \in(0,1]$ denotaremos con $\lambda_{1}(s)$ el primer punto fijo de $B_{s}(\lambda)$.

Lema 2.5. Si $\gamma$ satisface (A1)-(A3), entonces $\lambda_{1}(s)$ es estrictamente decreciente y continua.

Demostración. Supongamos que $s_{1}<s_{2}$. Del Teorema 1.1 deducimos que $B_{s_{2}}(\lambda)<B_{s_{1}}(\lambda)$ para todo $\lambda>0$. En particular para $\lambda_{1}=\lambda_{1}\left(s_{1}\right)$ y $\lambda_{2}=\lambda_{1}\left(s_{2}\right)$ se tiene que $\lambda_{2}<B_{s_{2}}\left(\lambda_{1}\right)<B_{s_{1}}\left(\lambda_{1}\right)=\lambda_{1}$.

Supongamos ahora que $\bar{s} \in(0,1],\left\{s_{n}\right\}_{n=1}^{\infty}$ es una sucesión en $(0,1], s_{n} \rightarrow \bar{s}$, y $\lambda_{1}\left(s_{n}\right) \rightarrow d$. Para cada $n$, existe $\vec{u}_{s_{n}} \in \mathbb{E}^{m} \cap \operatorname{Int} \mathbb{P}$ tal que $\left\|\vec{u}_{s_{n}}\right\|_{\infty}=1$, y $\widetilde{L}\left[\vec{u}_{s_{n}}\right]+\lambda_{1}\left(s_{n}\right) \gamma^{-} \vec{u}_{s_{n}}=\lambda_{1}\left(s_{n}\right) \rho_{s_{n}} \vec{u}_{s_{n}}$ en $\Omega \times \mathbb{R}$. Con el mismo argumento usado en el Lema 2.3 obtenemos una función $\overrightarrow{v_{0}} \in \mathbb{E}^{m} \cap \operatorname{Int} \mathbb{P}$, tal que $\left\|\overrightarrow{v_{0}}\right\|_{\infty}=1, \mathrm{y}$

$$
\tilde{L}\left[\overrightarrow{v_{0}}\right]+d \gamma^{-} \overrightarrow{v_{0}}=d \rho_{s^{-}} \overrightarrow{v_{0}} \quad \text { en } \Omega \times \mathbb{R} .
$$


De (2.12) es claro que $d \geq \lambda_{1}(\bar{s})$. Si $d \neq \lambda_{1}(\bar{s}), B_{\bar{s}}(\lambda)$ tendría un segundo punto fijo $\lambda_{0}>\lambda_{1}(\bar{s})$. Sea $\overline{\bar{\lambda}} \in\left(\lambda_{1}(\bar{s}), \lambda_{0}\right)$, es claro que $B_{\bar{s}}(\overline{\bar{\lambda}})<$ $\overline{\bar{\lambda}}$ y puesto que $\lim _{n \rightarrow \infty} B_{s_{n}}(\overline{\bar{\lambda}})=B_{\bar{s}}(\overline{\bar{\lambda}})$, existe un entero positivo $N_{0}$ tal que $B_{s_{n}}(\overline{\bar{\lambda}})<\overline{\bar{\lambda}}<\lambda_{0}$ para todo $n \geq N_{0}$. Consecuentemente, $\lambda_{1}\left(s_{n}\right)<$ $\overline{\bar{\lambda}}<\lambda_{0}$, y entonces, $d=\lambda_{1}(\bar{s})<\overline{\bar{\lambda}}<\lambda_{0}$.

Lema 2.6. Sean $p \in \mathbb{F} y \xi(t)=\max _{\Omega} p(x, t)$. El problema

$$
L[u]=\lambda p u \text { en } \Omega \times \mathbb{R}, u \in \mathbb{E}, u>0, \text { en } \Omega \times \mathbb{R}, \lambda>0,
$$

tiene solución si $\int_{0}^{\ell} \xi(t) d t>0$ y no tiene solución si $\int_{0}^{\ell} \xi(t) d t=0$.

Demostración. Si $\int_{0}^{\ell} \xi(t) d t>0$, la existencia de soluciones de (2.13) es consecuencia directa del Lema 2.4, puesto que es posible construir una función $\sigma: \mathbb{R} \rightarrow \Omega$ de clase $C^{2}$ tal que $\int_{0}^{\ell} p(\sigma(t), t) d t>0$.

Supongamos que $\int_{0}^{\ell} \xi(t) d t=0$ y que existe $\left(\bar{\lambda}, u_{\bar{\lambda}}\right)$ solución de (2.13), $\bar{\lambda}>0, u_{\bar{\lambda}}>0$. Sea $\phi_{0} \in \mathbb{E},\left\|\phi_{0}\right\|_{\infty}=1$, la solución del problema

$$
L[\phi]=\lambda_{0} \phi \quad \text { en } \Omega \times \mathbb{R}, \lambda_{0}>0, \phi>0, \phi \in \mathbb{E},\|\phi\|_{\infty}=1,
$$

(cf. [6]). Sea

$$
v_{\bar{\lambda}}=\phi(x, t) e^{\bar{\lambda} \int_{0}^{t} \xi(s) d s}
$$

entonces

$$
L\left[v_{\bar{\lambda}}\right]=\lambda_{0} v_{\bar{\lambda}}+\bar{\lambda} \xi(t) v_{\bar{\lambda}} .
$$

Puesto que $\xi(t) \geq p(x, t)$, el Teorema 1.1 implica que $\bar{\lambda} \xi(t)+\lambda_{0}=$ $\bar{\lambda} p(x, t)$. Esta contradicción demuestra el lema.

Denotemos con $\bar{\xi}(t)=\max \left\{\gamma_{k k}(x, t): x \in \Omega\right\}$. Por (1.6),

$$
\int_{0}^{\ell} \bar{\xi}(t) d t>0 \text {. }
$$

Sea $s^{*} \in(0,1)$, tal que

$$
s^{*} \int_{0}^{\ell} \bar{\xi}^{+}(t) d t=\int_{0}^{\ell} \bar{\xi}(t) d t
$$


Para cada $s \in(0,1], \quad \lambda>0$ existen $u_{\lambda} \in \mathbb{E}, u_{\lambda}>0$ y $\bar{B}_{s}(\lambda)>0$ tales que

$$
L\left[u_{\lambda}\right]+\lambda \gamma_{k k}^{-} u_{\lambda}=\bar{B}_{s}(\lambda)\left(s \gamma_{k k}^{+}\right) u_{\lambda} \quad \text { en } \Omega \times \mathbb{R} .
$$

Por el Lema anterior si $s \in\left(s^{*}, 1\right], \bar{B}_{s}(\lambda)$ tiene por lo menos un punto fijo, el primero de estos será denotado $\bar{\lambda}_{1}(s)$. Como en el Lema 2.5 se prueba que $\bar{\lambda}_{1}(s)$ es estrictamente decreciente y continua en $\left(s^{*}, 1\right]$.

Lema 2.7. $\bar{\lambda}_{1}(s) \rightarrow \infty$ cuando $s \rightarrow s^{*}$ por la derecha.

Demostración. Supongamos que $\bar{\lambda}_{1}(s) \rightarrow d<\infty$ cuando $s \rightarrow s^{*}, s>$ $s^{*}$. Sea $u_{s} \in \mathbb{E}, u_{s}>0$ en $\Omega \times \mathbb{R},\left\|u_{s}\right\|_{\infty}=1$, tal que

$$
L\left[u_{s}\right]+\bar{\lambda}_{1}(s) \gamma_{k k}^{-} u_{s}=\bar{\lambda}_{1}(s) s \gamma_{k k}^{+} u_{s} \quad \text { en } \Omega \times \mathbb{R} .
$$

Con el mismo argumento del Lema 2.3, existe $v \in \mathbb{E}, v>0$ en $\Omega \times \mathbb{R}$ tal que

$$
L[v]+d \gamma_{k k}^{-} v=d s^{*} \gamma_{k k}^{+} v \quad \text { en } \Omega \times \mathbb{R},
$$

lo cual contradice el lema anterior.

Lema 2.8. Si $(\lambda, \vec{u}) \in\left(\mathbb{R}_{+}-\{0\}\right) \times\left(\mathbb{E}^{m} \cap \operatorname{Int} \mathbb{P}\right)$ satisface (1.1), entonces $\lambda \leq \bar{\lambda}_{1}(1)$.

Demostración. Sea $\vec{u}=\operatorname{col}\left(u^{1}, \ldots, u^{m}\right)$, es claro que

$$
L\left[u^{k}\right] \geq \lambda\left[\gamma_{k k}^{+}-\gamma_{k k}^{-}\right] u^{k}
$$

Si suponemos que $\lambda>\bar{\lambda}_{1}(1)$, por el lema anterior, existen $s \in$ $\left(s^{*}, 1\right)$ y $u_{s} \in \mathbb{E}, u_{s}>0$, tales que $\lambda=\bar{\lambda}_{1}(s)$ y

$$
L\left[u_{s}\right]=\lambda\left[\gamma_{k k}^{+}-\gamma_{k k}^{-}\right] u_{s} \quad \text { en } \Omega \times \mathbb{R} .
$$

Entonces, por el Teorema 1.1, obtenemos una contradicción.

Lema 2.9. $\lambda_{1}(s) \rightarrow \bar{d} \geq \bar{\lambda}_{1}(1)$ si $s \rightarrow 0,(\bar{d} \leq \infty)$.

Demostración. Suponiendo que $\lambda_{1}(s) \rightarrow \bar{d}<\infty$, cuando $s \rightarrow 0$, como en los lemas 2.7 y 2.3 , obtenemos una función $\vec{v} \in \mathbb{E}^{m} \cap$ Int $\mathbb{P}$, 
$\frac{\vec{v}}{\lambda}=\operatorname{col}\left(v^{1}, \ldots, v^{m}\right)$, tal que $L\left[v^{k}\right]=d \gamma_{k k} v^{k}$ en $\Omega \times \mathbb{R}$. Entonces $\bar{d} \geq$ $\bar{\lambda}_{1}(1)$.

Demostración del Teorema 1.2. El Lema 2.4 y el Principio del Máximo implican la existencia de $\left(\lambda, \vec{u}_{1}\right) \in \mathbb{R}_{+} \times \mathbb{E}^{m} \cap \operatorname{Int} \mathbb{P}$, el cual satisface i) y ii), $\lambda_{1}=\lambda_{1}(1)$.

Sea $\left(\lambda, \vec{u}_{\lambda}\right) \in \mathbb{R}_{+} \times \mathbb{E}^{m} \cap \operatorname{Int} \mathbb{P}$, una solución de (1.1), con $\lambda>$ $\lambda_{1}(1)$. Por el Lema $2.8, \lambda \leq \bar{\lambda}_{1}(1)$, y por los Lemas 2.5 y 2.9 , existen $s \in(0,1)$ y $\vec{u}_{s} \in \mathbb{E}^{m} \cap \operatorname{Int} \mathbb{P}$ tales que $\lambda=\lambda_{1}(s), \mathrm{y}$

$$
\widetilde{L}\left[\vec{u}_{s}\right]=\lambda\left[s \gamma^{+}-\gamma^{-}\right] \vec{u}_{s} \quad \text { en } \Omega \times \mathbb{R} .
$$

Entonces, del Teorema 1.1, obtenemos que $s \gamma^{+}=\gamma^{+}$. Esta contradicción demuestra la unicidad de $\lambda_{1}$. La simplicidad de $\lambda_{1}$ es consecuencia de un resultado de Teoría de Perturbaciones Analíticas debido a Kato, (cf. [4, Lema 8.a]).

\section{Aplicaciones.}

En esta sección aplicaremos los teoremas 1.1 y 1.2 al estudio del sistema (1.2). En la demostración del Teorema 1.3 utilizaremos los siguientes lemas.

Lema 3.1. Si $u \in C^{2+\alpha, 1+(\alpha / 2)}\left(\bar{D}_{k} \times \mathbb{R}\right), f \in C^{\alpha, \alpha / 2}\left(\bar{D}_{k} \times \mathbb{R}\right), f(x, t+$ $\ell) \equiv f(x, t), u(x, t+\ell) \equiv u(x, t)$ y $L[u] \equiv f$ en $D_{k} \times \mathbb{R}$, entonces

$$
\|u\|_{2+\alpha}^{D_{k} \times[0, \ell]} \leq k\left[\|u\|_{\alpha+1}^{\partial D_{k} \times[0, \ell]}+\|u\|_{\alpha}^{D_{k} \times[0, \ell]}+\|f\|_{\alpha}^{D_{k} \times[0, \ell]}\right]
$$

$\left(D_{k}\right.$ está dado en $\left.\left(C^{*}\right)\right)$.

Demostración. Sea $\phi \in C^{\infty}(\mathbb{R})$, tal que $\phi(\ell / 2)=0, \phi(t)=1$ para $t \geq \ell, 0 \leq \phi \leq 1$ en $\mathbb{R}$. Es claro que

$$
L[\phi u]=\phi^{\prime} u+\phi f \quad \text { en } D_{k} \times \mathbb{R},
$$

para todo $k, \mathrm{y}$

$$
\|u\|_{2+\alpha}^{D_{k} \times[0, \ell]}=\|u\|_{2+\alpha}^{D_{k} \times[\ell, 2 \ell]}=\|\phi u\|_{2+\alpha}^{D_{k} \times[\ell, 2 \ell]} \leq\|\phi u\|_{2+\alpha}^{D_{k} \times[\ell / 2,2 \ell]} .
$$


Entonces, a partir de una desigualdad clásica en la teoría de ecuaciones parabólicas ( $c f$. [6, Cap IV, Teorema 5.3]), obtenemos

$$
\begin{aligned}
\|\phi u\|_{2+\alpha}^{D_{k} \times[\ell / 2, \ell]} & \leq c_{1}\left(\|\phi u\|_{1+\alpha}^{\partial D_{k} \times[\ell / 2, \ell]}+\left\|\phi f+\phi^{\prime} u\right\|_{\alpha}^{D_{k} \times[\ell / 2,2 \ell}\right) \\
& \leq k_{3}\left(\|u\|_{1+\alpha}^{\partial D_{k} \times[0, \ell]}+\|u\|_{\alpha}^{D_{k} \times[0, \ell]}+\|f\|_{\alpha}^{D_{k} \times[0, \ell]}\right)
\end{aligned}
$$

ObSERVACIón 3.1. Sean $\widehat{\mathbb{F}}=\{\hat{\boldsymbol{u}} \in C(\bar{\Omega} \times \mathbb{R})$ : existe una sucesión $\left\{u_{n}\right\}$ en $\mathbb{F}$ tal que $\left.\left\|u_{n}-\hat{u}\right\|_{\infty} \rightarrow 0\right\},\|\cdot\|_{\widehat{F}}=\|\cdot\|_{\infty}$, y $\widetilde{\mathbf{F}}$ como en los preliminares.

Por el Lema 2.2, el operador $L^{-1}: \mathbb{F} \rightarrow \mathbb{E}$ puede ser extendido por continuidad al operador continuo y positivo $\widehat{L}^{-1}: \widehat{\mathbf{F}} \rightarrow \widetilde{\mathbf{F}}$, (se define $\widehat{L}^{-1}(\hat{u})=\hat{v}$ si y sólo si $\hat{v}=\lim _{n \rightarrow \infty}\left(L^{-1}\left(u_{n}\right)\right)$ en la $\widetilde{\mathbf{F}}$-norma, donde $\left\{u_{n}\right\}$ está en $\mathbb{F}$ y $\left\|u_{n}-\hat{u}\right\|_{\infty} \rightarrow 0$ )

El siguiente Lema es muy conocido ( $c f .[1])$.

Lema 3.2. Sea $g(x, t, u)$ una función continua definida de $\overline{\boldsymbol{\Omega}} \times \mathbb{R} \times \mathbb{R}$ en $\mathbb{R}$, $\ell$-periódica en $t$, tal que $\partial g / \partial u$ es continua y $g(\cdot, \cdot, v)$ y $\partial g(\cdot, \cdot, v) / \partial u$ pertenecen a $\mathbb{F}$, para todo $v \in \mathbb{F}$. Si existen dos funciones $v, w \in \mathbb{F} \cap$ $C^{2, o}(\bar{\Omega} \times \mathbb{R})$ tales que

$$
L[v] \geq g(\cdot, \cdot, v), \quad L[w] \leq g(\cdot, \cdot, w)
$$

$w \leq v$ en $\Omega \times \mathbb{R}, v \geq 0 \geq w$ en $\partial \Omega \times \mathbb{R}$, entonces existen dos soluciones $u^{*}, u_{*} \in \mathbb{E}$ del problema

$$
L[u]=g(\cdot, \cdot, u) \quad \text { en } \Omega \times \mathbb{R}, u \in \mathbb{E}
$$

tales que

i) $w \leq u_{*} \leq u^{*} \leq v$ en $\bar{\Omega} \times \mathbb{R}, y$

ii) si ũ es solución de (3.3) y $w \leq \tilde{u} \leq v$ en $\Omega \times \mathbb{R}$, entonces $u_{*} \leq$ $\tilde{u} \leq u^{*}$ en $\Omega \times \mathbb{R}$.

(Dos funciones $v, w$ que satisfacen (3.2) se llaman supersoluciones y subsoluciones de (3.3) respectivamente). 
Lema 3.3. Si se satisface (B2), para cada $\vec{u}=\operatorname{col}\left(u^{1}, \ldots, u^{m}\right) \in \mathbb{P}$, existe una única solución $T(\vec{u}) \in \mathbb{E}, T(\vec{u}) \geq 0$ en $\Omega \times \mathbb{R}$ del problema

$$
L[T(\vec{u})]=-C(x, t) T(\vec{u})(x, t)+\sum_{j=1}^{m} G_{j}(T(\vec{u}), x, t) u^{j} \text { en } \Omega \times \mathbb{R}
$$

Demostración. Sean $\vec{u}=\operatorname{col}\left(u^{1}, \ldots, u^{m}\right) \in \mathbb{P}, \underline{T}(x, t) \equiv 0$ y $\bar{T}(x, t)$ $\equiv k$ en $\Omega \times \mathbb{R}$, con $k$ suficientemente grande tal que

$$
\begin{aligned}
L[\bar{T}]=-\bar{c}_{0} k \geq 0 \geq & -C(x, t) \bar{T}(x, t) \\
& +\sum_{j=1}^{m} G_{j}(\bar{T}, x, t) u^{j} \quad \text { en } \Omega \times \mathbb{R} .
\end{aligned}
$$

$\underline{T}$ y $\bar{T}$ son una subsolución y supersolución de (3.4) respectivamente. Entonces, por el lema anterior, existe una solución $T \in \mathbb{E}, T \geq 0$ de (3.4).

La unicidad de la solución también es consecuencia del lema anterior. Supongamos que $T_{1}$ y $T_{2}$ son dos soluciones de (3.4). Tomemos $T(x, t)=k \geq T_{1}, T_{2} \geq 0$ en $\bar{\Omega} \times \mathbb{R}$, y $\underline{T}(x, t) \equiv 0$. Por el Lema 3.2 , existen dos soluciones $T^{*}, T_{*}$ de (3.4) tales que $\underline{T} \leq T_{*} \leq T_{i} \leq T^{*} \leq \bar{T}$ en $\bar{\Omega} \times \mathbb{R}, i=1,2$. Puesto que

$$
L\left[T_{*}-T^{*}\right]=C(\cdot)\left(T^{*}-T_{*}\right)+\sum_{j=1}^{m}\left(G_{j}\left(T_{*}, \cdot\right)-G_{j}\left(T^{*}, \cdot\right)\right) u^{j} \geq 0
$$

en $\Omega \times \mathbb{R}$, el Principio del Máximo implica que $T_{*}=T^{*}$.

El problema (1.2) es equivalente a

$$
\vec{u}=\mathbb{K}[\vec{u}]+G[\vec{u}], \quad \vec{u} \in \widehat{\mathbb{E}}
$$

donde

$$
\mathbb{K}[\vec{u}]=(\widetilde{L})^{-1}(H(0, \cdot) \vec{u})
$$

y

$$
G[\vec{u}]=(\widetilde{L})^{-1}([H(T(\vec{u}), \cdot)-H(0, \cdot)] \vec{u}) .
$$

Consideraremos el problema auxiliar

$$
[I-\lambda\urcorner] \vec{u}+\lambda(-G[\vec{u}])=0, \quad(\lambda, \vec{u}) \in \mathbb{R}_{+} \times \widehat{\mathbb{E}} .
$$


Por el Teorema 1.2, $\mathbb{K}$ tiene un valor característico $\widehat{\lambda}_{0}$ con función propia correspondiente $\vec{\psi}_{0}=\operatorname{col}\left(\psi_{0}^{1}, \ldots, \psi_{0}^{m}\right) \in \mathbb{E}^{m},\left(\widehat{\lambda}_{0}, \vec{\psi}_{0}\right)$ satisface (ii) y (iii) del mismo teorema. Puesto que

$$
\frac{\|-\lambda G(\vec{u})\|_{\widehat{\mathbb{E}}}}{\|\vec{u}\|_{\widehat{\mathbb{E}}}} \leq \frac{\lambda k_{2}}{[\vec{u}]_{\infty}}\|[H(T(\vec{u}), \cdot)-H(0, \cdot)] \vec{u}\|_{\infty} \rightarrow 0, \text { si }\|\vec{u}\|_{\widehat{\mathbb{E}}} \rightarrow 0,
$$

por un resultado de la Teoría de Bifurcación (cf. [2, Teorema 29.1]), obtenemos el siguiente lema:

Lema 3.4. Si se satisfacen (B1)-(B2), entonces $\left(\widehat{\lambda}_{0}, \overrightarrow{0}\right)$ es un punto de bifurcación por soluciones positivas de (3.7) y la componente conexa $\mathcal{C}$ de $\bar{M} \subseteq \mathbb{R}_{+} \times \widehat{\mathbb{E}}$, la cual contiene a $\left(\widehat{\lambda}_{0}, \overrightarrow{0}\right)$ es no acotada, donde $M=\left\{(\lambda, \vec{u}) \in \mathbb{R}_{+} \times \mathbb{P}: \vec{u} \not \equiv \overrightarrow{0}\right.$ y $(\lambda, \vec{u})$ es solución de $\left.(3.7)\right\}$.

La bifurcación es por soluciones positivas debido a que $\vec{\psi}_{0}$ pertenece al interior de $\mathbb{P}$.

Si $\Omega$ satisface $\left(C^{*}\right)$, por el Teorema 1.2 , para cada entero positivo $k$, existen $d_{k}>0$ y $\vec{\phi}_{k}=\operatorname{col}\left(\phi_{k}^{1}, \ldots, \phi_{k}^{m}\right), \phi_{k}^{i} \in C^{2+\alpha, 1+(\alpha / 2)}\left(\bar{D}_{k} \times \mathbb{R}\right)$ tales que $\left(d_{k}, \vec{\phi}_{k}\right)$ satisface (ii) y (iii) del mismo teorema, $\left\|\vec{\phi}_{k}\right\|_{1+\sigma}^{\bar{D}_{k} \times[0, \ell]}=$ $1, \vec{\phi}_{k} \equiv \overrightarrow{0}$ en $\partial \Omega \times \mathbb{R}, \mathrm{y}$

$$
\begin{gathered}
\widetilde{L}\left(\vec{\phi}_{k}\right)=d_{k} H(0, \cdot)\left[\vec{\phi}_{k}\right] \quad \text { en } D_{k} \times \mathbb{R}, \\
\text { y } \quad \vec{\phi}_{k}(x, t+\ell)=\vec{\phi}_{k}(x, t),
\end{gathered}
$$

$$
\left(\sigma \in(\alpha, 1) \text { fijo, }\left\|\vec{\phi}_{k}\right\|_{1+\alpha}^{\bar{D}_{k} \times[0, \ell]}=\max \left\{\left\|\phi_{k}^{i}\right\|_{1+\alpha}^{\bar{D}_{k} \times[0, \ell]}, \quad i=1, \ldots, m\right\}\right) .
$$

Lema 3.5. Si $H(0, \cdot)$ satisface (A1)-(A3) y $\Omega$ satisface $\left(C^{*}\right)$, entonces $d_{k}>d_{k+1}$ para todo entero positivo $k, y d_{k} \rightarrow \widehat{\lambda_{0}}$.

Demostración. Por el Teorema 1.1, $d_{1}>\ldots>d_{k}>d_{k+1}>\ldots>\widehat{\lambda}_{0}$ y por lo tanto $d_{k} \rightarrow \hat{\lambda}_{1}$, para cierto $\hat{\lambda}_{1} \geq \hat{\lambda}_{0}$. Denotaremos con $\widetilde{\phi}_{k}^{i}$ la extensión por cero de $\phi_{k}^{i}$ a $\bar{\Omega} \times \mathbb{R}, \widetilde{\phi}_{k}=\operatorname{col}\left(\widetilde{\phi}_{k}^{1}, \ldots, \widetilde{\phi}_{k}^{m}\right)$. Por ser compacta la inclusión

$$
J^{*}: C^{\sigma, \sigma / 2}(\bar{\Omega} \times \mathbb{R}) \rightarrow C^{\alpha, \alpha / 2}(\bar{\Omega} \times \mathbb{R}),
$$

(para una subsucesión), $\widetilde{\phi}_{k} \rightarrow \vec{\psi}$ en $\mathbb{F}^{m}$, para un $\vec{\psi} \in \mathbb{F}^{m}$. 
Para todo entero positivo $s$ fijo, si $k \geq s$,

$$
1 \geq\left\|\vec{\phi}_{k}\right\|_{1+\sigma}^{\bar{D}_{s} \times[0, \ell]}
$$

Entonces (para una subsucesión) $\vec{\phi}_{k} \rightarrow \vec{\psi}$ en la $\|\cdot\|_{1+\alpha}^{\bar{D}_{s} \times[0, \ell]}$-norma y $\vec{\psi} \in C^{1+\alpha,(\alpha / 2)}\left(\bar{D}_{s} \times[0, \ell]\right)$. Puesto que

$$
\widetilde{L}\left(\vec{\phi}_{k}-\vec{\phi}_{n}\right)=d_{k} H(0, \cdot)\left[\vec{\phi}_{k}-\vec{\phi}_{n}\right]+\left(d_{k}-d_{n}\right) H(0, \cdot) \vec{\phi}_{n}
$$

end $D_{s} \times \mathbb{R}, k, n \geq s$, utilizando la desigualdad (3.1), obtenemos que

$$
\left\|\phi_{k}^{i}-\phi_{n}^{i}\right\|_{2+\alpha}^{\bar{D}_{\boldsymbol{s}} \times[0, \ell]} \rightarrow 0, \quad \text { cuando } n, k \rightarrow \infty .
$$

En consecuencia $\phi_{k}^{i} \rightarrow \psi^{i}$ en $C^{2+\alpha, 1+(\alpha / 2)}\left(\bar{D}_{s} \times[0, \ell]\right)$ y $\vec{\psi} \not \equiv \overrightarrow{0}$. Tomando límite en (3.8) obtenemos

$$
\widetilde{L}(\vec{\psi})=\widehat{\lambda}_{1} H(0, \cdot) \vec{\psi} \quad \text { en } D_{s} \times[0, l], \quad \text { para todo } s \geq 1 .
$$

Por lo tanto

$$
\widetilde{L}[\vec{\psi}]=\widehat{\lambda}_{1} H(0, \cdot) \vec{\psi} \quad \text { en } \Omega \times \mathbb{R}, \quad \vec{\psi} \in \mathbb{P}, \quad \vec{\psi} \not \equiv \overrightarrow{0} .
$$

Finalmente, por el Teorema 1.1, concluimos que $\widehat{\lambda}_{1}=\widehat{\lambda}_{0}$.

Observación 3.2. Si $H(0, \cdot)$ satisface (A1)-(A3) y (B3) b), entonces siguiendo el mismo razonamiento del Lema 2.4, podemos probar que el conjunto

$$
\begin{gathered}
\Lambda=\left\{\lambda>0: \text { existe } \vec{u}_{\lambda} \in \mathbb{E}^{m} \cap \mathbb{P}, \vec{u}_{\lambda} \not \equiv \overrightarrow{0}\right. \text { tal que } \\
(\lambda, \vec{u}) \text { es solución de }(3.7)\}
\end{gathered}
$$

es acotado.

Demostración del Teorema 1.3. Para probar la parte a) demostraremos que si se satisfacen (B1)-(B3) y $\Omega$ satisface $\left(C^{*}\right)$, entonces existe $\vec{u} \in \mathbb{P}$ tal que $(1, \vec{u}) \in M$.

Por la Observación 3.2, el conjunto $\{\lambda:(\lambda, \vec{u}) \in \mathcal{C}\}$ es acotado. Por el Lema 3.4, existe una sucesión $\left\{\left(\lambda_{n}, \vec{u}_{n}\right)\right\}$ en $M$ tal que $\left\|\vec{u}_{n}\right\|_{\widehat{\mathbb{E}}} \rightarrow \infty$, y $\lambda_{n} \rightarrow \bar{\lambda}$, para cierto $\bar{\lambda} \in \mathbb{R}, \vec{u}_{n}=\operatorname{col}\left(u_{n}^{1}, \ldots, u_{n}^{m}\right) \mathrm{y}$

$$
\widetilde{L}\left[\vec{u}_{n}\right]=\lambda_{n}\left[H\left(T\left(\vec{u}_{n}\right), \cdot\right)\right] \vec{u}_{n} \quad \text { en } \Omega \times \mathbb{R} .
$$


Probaremos primero que $T\left(u_{n}\right) \rightarrow \infty$, uniformemente sobre subconjuntos compactos de $\Omega \times \mathbb{R}$. Definimos las sucesiones

$$
z_{n}=\frac{T\left(\vec{u}_{n}\right)}{\left\|\vec{u}_{n}\right\|_{\widehat{\mathbb{E}}}} \quad \text { y } \quad \vec{w}_{n}=\frac{\vec{u}_{n}}{\left\|\vec{u}_{n}\right\|_{\widehat{\mathbb{E}}}}, \quad w_{n}=\operatorname{col}\left(w_{n}^{1}, \cdots, w_{n}^{n}\right) .
$$

Dividiendo (3.9) por $\left\|\vec{u}_{n}\right\|_{\widehat{\mathbb{R}}}$, por el Lema 2.2, obtenemos que (para una subsucesión) $\vec{w}_{n} \rightarrow \vec{w}$ en $\widehat{\mathbb{E}}$, para un $\vec{w} \in \widehat{\mathbb{E}}$. Análogamente, por ser acotado el lado derecho de

$$
L\left[z_{n}\right]=-C z_{n}+\sum_{j=1}^{m} G_{j}\left(T\left(\vec{u}_{n}\right), \cdot\right) w_{n}^{j} \quad \text { en } \Omega \times \mathbb{R},
$$

(para una subsucesión) $z_{n} \rightarrow z \in \widetilde{\mathbb{F}}$, para un $z \in \widetilde{\mathbb{F}}$. Para cada $\vec{w}_{n}$ (respectivamente, para $\vec{w}$ ) existe $v_{n} \in \mathbb{E}$ (respectivamente, $v \in \mathbb{E}$ ) tal que

$$
L\left[v_{n}\right]=-C v_{n}+\sum_{j=1}^{m} g_{j} w_{n}^{j}, \quad v_{n}>0, \quad \text { en } \Omega \times \mathbb{R},
$$

(respectivamente, $L[v]=-C v+\sum_{j=1}^{m} g_{j} w^{j}, v>0$, en $\Omega \times \mathbb{R}$ ). Es fácil ver que (para una subsucesión), $v_{n} \rightarrow v$ en $\widetilde{\mathbb{F}}, v_{n} \leq z_{n}$, y por lo tanto, $z \geq v$ en $\Omega \times \mathbb{R}$. Puesto que

$$
z_{n}=\frac{T\left(\vec{u}_{n}\right)}{\left\|\vec{u}_{n}\right\|_{\widehat{\mathbb{E}}}}
$$

concluimos que

$$
T(\vec{u}) \rightarrow \infty \text { si } n \rightarrow \infty
$$

uniformemente sobre conjuntos compactos de $\Omega \times \mathbb{R}$.

Por el Lema 3.5, existe un entero $r$ tal que $a_{0}>d_{r}>\widehat{\lambda}_{0}$, y por (3.10) y (B3), existe $n_{0}$ tal que si $n \geq n_{0}$, entonces

$$
H_{i j}\left(T\left(\vec{u}_{n}\right)(x, t), x, t\right) \geq a_{0} H_{i j}(0, x, t) \quad \text { en } \bar{D}_{r} \times[0, \ell] .
$$

Puesto que

$$
\widetilde{L}\left[\vec{u}_{n}\right]=\lambda_{n} H\left(T\left(\vec{u}_{n}\right), \cdot\right) \vec{u}_{n}, \quad \text { en } D_{r}[0, \ell],
$$


y

$\widetilde{L}\left[\vec{\phi}_{r}\right]=d_{r} H(0, \cdot) \vec{\phi}_{r}, \quad$ en $D_{r} \times[0, \ell], \quad \vec{\phi}_{r} \equiv \overrightarrow{0}, \quad$ en $D_{r}[0, \ell]$,

(3.11) y el Teorema 1.1 implican que $\lambda_{n} \leq d_{r} / a_{0}<1$, para todo $n \geq n_{0}$. De la conexión de $\mathcal{C}$ se sigue el lema.

La parte b) se demuestra en forma similar.

\section{Referencias.}

[1] Amann, H., Periodic solutions of semilinear parabolic equations, in Nonlinear Analysis, volume in honor of E. H. Rothe, Academic Press (1978), $1-9$.

[2] Deimling, K., Nonlinear functional analysis. Springer-Verlag, 1985.

[3] Friedman, A., Partial differential equations of parabolic type. Prentice Hall, 1964.

[4] Hess, P. and Kato, T., On some linear and non-linear eigenvalue problems with an indefinite weight function. Comm. P. D. E. 5 (1980), 999-1030.

[5] Krasnoselskii, M., Positive solutions of operator equations. Noordhoff, 1964.

[6] Ladyzenskaja, O.A., Solonnikov, V.A. and Uralceva, N.N., Linear and quasi-linear equations of parabolic type. Translations of Math. Monographs, Amer. Math. Soc., 1968.

[7] Lazer, A.C., Some remarks on periodic solutions of parabolic differential equations, in Dynamical Systems, II. Bednarek-Cesari eds., Academic Press (1982), 227-246.

[8] Leung, A. and Chen, G.S., Elliptic and parabolic systems for neutron fission and diffusion. J. Math. Anal. Appl. 120 (1986), 655-669.

[9] Leung, A. and Gen-Shun Chen., Positive solutions for temperaturedependent two-group neutron flux equations: Equilibria and stabilities. SIAM J. Math. Anal 15 (1984), 490-499.

[10] Manes, A. and Micheletti, A., Una extensione della teoria variazionale classica degli autovalori per operatori ellittici del secondo ordine. Bolletino U.M.I. 7 (1973), 285-301.

[11] Ortega, L., Sobre las soluciones periódicas del problema de Dirichleț para ecuaciones de tipo parabólico. Rev. Colombiana de Matemáticas XIX (1985), 233-250. 
[12] Protter, M. and Weinbergger, H., Maximum principles in differential equations. Prentice Hall, 1967.

[13] Smulev, I., Periodic solutions of the first boundary problem for parabolic equations. Trans. Amer. Math. Soc. 79 (1969), 216-229,

Recibido: 24 de mayo de 1.991

Beatriz Elena Villa

Departamento de Matemáticas

Universidad Nacional de Colombia

Bogotá, COLOMBIA 